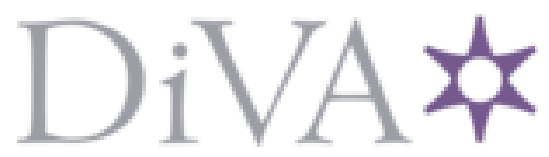

http://www.diva-portal.org

\title{
Postprint
}

This is the accepted version of a paper published in Journal of Physics B: Atomic, Molecular and Optical Physics. This paper has been peer-reviewed but does not include the final publisher proof-corrections or journal pagination.

Citation for the original published paper (version of record):

Nagaya, K., Sugishima, A., Iwayama, H., Murakami, H., Yao, M. et al. (2013)

Unusual under-threshold ionization of neon clusters studied by ion spectroscopy.

Journal of Physics B: Atomic, Molecular and Optical Physics, 46(16 SI): 164023

http://dx.doi.org/10.1088/0953-4075/46/16/164023

Access to the published version may require subscription.

N.B. When citing this work, cite the original published paper.

Permanent link to this version:

http://urn.kb.se/resolve?urn=urn:nbn:se:uu:diva-2 10259 


\section{Unusual Under-threshold Ionization of Neon Clusters Studied by Ion Spectroscopy}

K. Nagaya, ${ }^{1,2, *}$ A. Sugishima, ${ }^{1,2}$ H. Iwayama, ${ }^{1,2}$ H. Murakami, ${ }^{1,2}$ M. Yao, ${ }^{1,2}$ H. Fukuzawa, ${ }^{2,3}$ X.-J. Liu, ${ }^{2,3}$ K. Motomura,,${ }^{2,3}$ K. Ueda, ${ }^{2,3}$ N. Saito, ${ }^{2,4}$ L. Foucar, ${ }^{2,3,5}$ A. Rudenko, ${ }^{2,6}$ M. Kurka, ${ }^{2,3,7}$ K.-U. Kühnel, ${ }^{2,7}$ J. Ullrich, ${ }^{2,7,8}$ A. Czasch, ${ }^{9}$ R. Dörner, ${ }^{9}$ R. Feifel, ${ }^{10}$ M. Nagasono,${ }^{2}$ A. Higashiya,${ }^{2}$ M. Yabashi, ${ }^{2}$ T. Ishikawa, ${ }^{2}$ T. Togashi, ${ }^{2,11}$ H. Kimura, ${ }^{2,11}$ and H. Ohashi ${ }^{2,11}$

${ }^{1}$ Department of Physics, Graduate School of Science, Kyoto University, Kyoto 606-8502, Japan ${ }^{2}$ RIKEN, XFEL Project Head Office, Hyogo 679-5148, Japan

${ }^{3}$ Institute of Multidisciplinary Research for Advanced Materials, Tohoku University, Sendai 980-8577, Japan

${ }^{4}$ National Metrology Institute of Japan, AIST, Tsukuba 305-8568, Japan ${ }^{5}$ Max-Planck-Institut fuer Medizinische Forschung, Jahnstraße 29, 69120 Heidelberg, Germany

${ }^{6}$ J. R. Macdonald Laboratory, Department of Physics, Kansas State University, Manhattan KS 66506, USA

${ }^{7}$ Max-Planck-Insitut für Kernphysik, D-69117 Heidelberg, Germany ${ }^{8}$ Physikalisch-Technische Bundesanstalt, D-38116 Braunschweig, Germany ${ }^{9}$ Institut für Kernphysik, Universität Frankfurt, D-60486 Frankfurt, Germany ${ }^{10}$ Department of Physics and Astronomy, Uppsala University, SE-751 20 Uppsala, Sweden

${ }^{11}$ Japan Synchrotron Radiation Research Institute, Hyogo 679-5198, Japan (Dated: February 26, 2013) 


\begin{abstract}
We carried out time-of-flight mass spectrometry for neon clusters that were exposed to intense free electron laser pulses with the wavelength of $62 \mathrm{~nm}$, which induce optical transition from the ground state $\left(2 s^{2} 2 p^{6}\right)$ to an excited state $\left(2 s^{2} 2 p^{5} n l\right)$ in the Ne atoms. In contrast to $\mathrm{Ne}^{+}$ions produced by two-photon absorption from isolated $\mathrm{Ne}$ atoms, the $\mathrm{Ne}^{+}$ion yield from Ne clusters shows a linear dependence on the laser intensity $(I)$. Combining the results of our recent electron spectroscopy experiments, we suggest that the linear behavior with respect to $I$ is due to the nano-plasma that is formed as a result of Exciton-Mott Transition.
\end{abstract}

PACS numbers: $36.40 .-\mathrm{c}, 32.80 . \mathrm{Rm}$ 


\section{INTRODUCTION}

"More is different" is a famous phrase given by P.W. Anderson [1], who proposed that, as the number of constituent atoms increases, new phenomena could emerge. In the present work, we investigate ionization processes of clusters irradiated by free electron laser (FEL) pulses. Here, "More is different" has two aspects: we can control not only the number of constituent atoms but also the number of photons over a wide range. Multi-photon absorption by a single atom, which leads to the production of a highly charged ion, is a wellknown example for varying the number of photons, while the shift of the ionization potential with cluster size is an example for varying the number of atoms e.g. for the single-photon absorption. An particularly intriguing situation is encountered when a cluster is exposed to photons whose energy is lower than the ionization potential IP. In this case, if any ionization event (i.e. under-threshold ionization) takes place, it must be an effect of "More is different", either due to the number of photons or to the number of atoms. Furthermore, if the photon energy is tuned to the Rydberg states or exciton levels, various auto-ionization processes such as Interatomic Coulombic Decay (ICD) [2, 3] and other phenomena like the Exciton-Mott Transition (EMT) [4] are expected to occur. ICD in general is induced by two-center energy transfer, and conventionally it is triggered by ionizing an inner-valence electron. Recently, Kuleff et al. [5] proposed a novel ICD mechanism, in which two electrons are photo-excited from outer valence orbitals to the Rydberg states and one of them is then emitted by using the relaxation energy from the other. EMT is defined as an insulator-to-metal transition, in which the electron-hole correlation plays a crucial role: an exciton gas that was created by optical means can be transformed to an electron-hole plasma when the exciton density is high enough to induce screening effects by overlapping of the wave functions.

In this study we adopted neon clusters with an average size $\langle N\rangle$ of 1000 atoms, and exposed them to extreme ultraviolet free electron laser (EUV-FEL) pulses with wavelength of $62 \mathrm{~nm}$, which corresponds to the optical transition from the ground state $\left(2 s^{2} 2 p^{6}\right)$ to an excited state $\left(2 s^{2} 2 p^{5} n l\right)$ in a Ne atom [6]. We found that, whereas the $\mathrm{Ne}^{+}$ion yield from uncondensed Ne gas shows a quadratic dependence on the laser intensity $(I)$, indicating two-photon absorption, the $\mathrm{Ne}^{+}$ion yield from Ne clusters shows a linear dependence on the laser intensity. This work was followed by a series of electron spectroscopy experiments by our group, and some of the information on the electronic states is used in the following 
sections. However, as the experimental results are concerned, we restrict ourselves to ion spectroscopy in this paper.

\section{EXPERIMENT}

Experiments were performed at SPring-8 Compact SASE Source (SCSS) test accelerator in Japan [7]. Our experimental setup was almost the same as the one reported in [8, 9]. Briefly, the cluster beam crossed the FEL beam at 45 degrees in the horizontal plane. The photon energy was tuned to $20 \mathrm{eV}(62 \mathrm{~nm})$. The FEL beam was partially blocked by a $1.5 \mathrm{~mm}$ wide horizontal beam stopper before the ionization region so that the unfocused beam did not irradiate the cluster beam directly. The FEL beam was back focused onto the cluster beam by a multi-layer focusing mirror fabricated at Lawrence Berkeley National Laboratory, which was the same one used in [8]. Taking all the optical elements (deflecting and focusing mirrors, etc) between the radiation source point and the ionization volume into account, we estimated the power density at the focus spot on the cluster beam to be at most $\sim 10^{14} \mathrm{~W} / \mathrm{cm}^{2}$ at full power of the FEL, assuming a diffraction limited focus size of $3 \mu \mathrm{m}$ in diameter and a pulse length of 100 fs.

The cluster beam was prepared by adiabatic expansion of Ne gas through a pulsed $250 \mu \mathrm{m}$ nozzle. The stagnation pressure was 4.6 bar, and the nozzle temperature was $80 \mathrm{~K}$ [10]. The average cluster sizes $\langle N\rangle$ was estimated to be 1000 atoms according to scaling laws [11, 12]. To avoid space-charge effects due to ionization of background gases, the pulsed gas jets was cut to $0.6 \mathrm{~mm}$ width and $0.4 \mathrm{~mm}$ height with knife-edge slits and traveled to the focus spot located at $1.7 \mathrm{~m}$ downstream from the nozzle.

We measured time-of-flight (TOF) spectra with our momentum imaging spectrometer $[8,9]$. Fragment ions were vertically extracted by a uniform electrostatic field. They travel through an extraction region ( $75 \mathrm{~V} / \mathrm{cm}$ electric field strength, $40 \mathrm{~mm}$ in length), an acceleration region $(110 \mathrm{~V} / \mathrm{cm}$ electric field strength, $52 \mathrm{~mm}$ in length) and a field-free region (308 mm), and are finally detected by microchannel-plate (MCP) detectors equipped with a three-layer delay-line anode (RoentDek HEX120) [13].

The measurements were done with the full laser power and with one third of it. In the latter case, the intensity was attenuated by transmitting the laser pulses through a gas chamber filled with Ar gas. In practice, however, since the laser ejected from the SASE source 
fluctuates shot by shot, we measured the intensity of each pulse by a gas monitor detector (GMD). At the same time, the TOF spectrum was also measured for each laser shot. Both signals were recorded by an 8-channel digitizer (Acqiris DC282×2), and the timing signals were extracted by a software constant fraction discriminator [14]. This procedure enabled us to deduce precise laser-power dependences of the TOF spectra.

\section{RESULTS}

In Fig. 1, the spectrum (a) displays the TOF spectrum accumulated during the experiment with $1 / 3$ of the full power. The corresponding power distribution is shown as dark shaded area in the inset of Fig. 1 as a function of the GMD channel. For the $1 / 3$ power, the weighted average of the GMD channel is 1102. Since the power distribution is much wider for the full power experiment, we have divided the distribution into three parts: (b) below 2980 channel, (c) from 3000 to 3360 channels, and (d) above 3380 channel (the grey, light grey and white distribution). The average channel number is 2547 for (b), 3168 for (c) and 3639 for (d). In each corresponding TOF spectrum a prominent peak is observed at the mass-to-charge ratio $m / q$ of 20 , and a smaller one at $m / q=22$, corresponding to ${ }^{20} \mathrm{Ne}^{+}$and its isotope ${ }^{22} \mathrm{Ne}^{+}$, respectively. In addition, broad distributions centered at $m / q=20$ and $m / q=22$ are also seen. We believe that the broad distributions resemble fragments from clusters whereas the sharp peaks correspond to ions from uncondensed gas, because large fragment energies resulting in a broad TOF distribution can only be produced by Coulomb repulsion between ions in clusters. No multiply charged ions such as $\mathrm{Ne}^{2+}$ were observed.

We now examine the laser intensity dependence of ion yield to clarify the multiphoton ionization mechanism. We aim to find out if the ionization proceeds via a sequence of single photon absorption steps at different Ne sites or if two photons are absorbed at a single site. We decomposed the TOF spectra into a sum of two narrow and two wide Lorentzian curves, as displayed in the lower panel of Fig. 1. The height and width parameters were determined by a least square fitting. In the fitting procedure, however, the $m / q$ region below 20 was eliminated, because the MCP signal in this region was distorted due to $\mathrm{H}_{2} \mathrm{O}$ contamination, which we know from the fact that the apparent ratio of ${ }^{20} \mathrm{Ne}^{+}$to ${ }^{22} \mathrm{Ne}^{+}$for uncondensed atoms deviates progressively from the natural abundance (c.a. 9:1) with increasing laser power. Thus, we use the data for ${ }^{22} \mathrm{Ne}^{+}$hereafter. The benefit of the ${ }^{22} \mathrm{Ne}^{+}$isotope was 

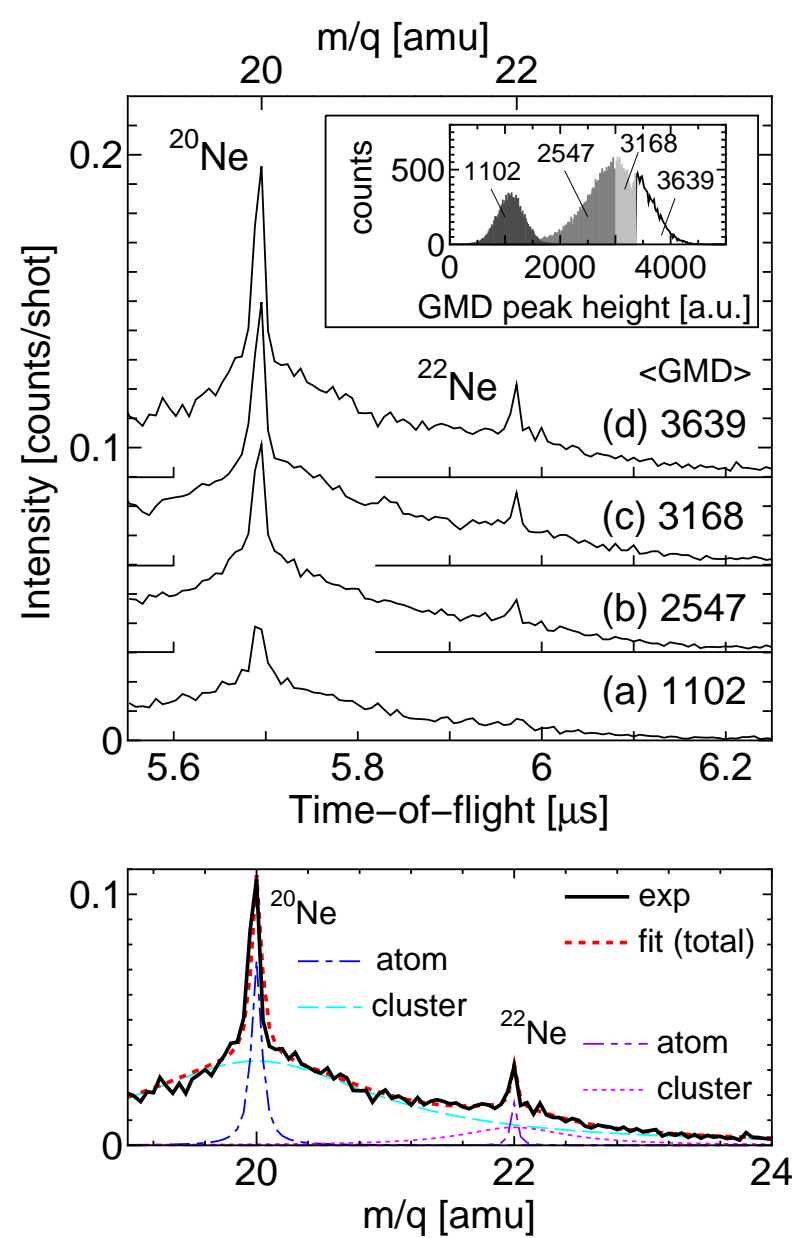

FIG. 1: Upper panel: TOF spectra of a $\mathrm{Ne}_{1000}$ cluster beam irradiated by $62 \mathrm{~nm}$ FEL pulses. The numbers in figure denote the channel numbers of the gas monitor detector (GMD). Inset shows the power distribution in the GMD for the full and for $1 / 3$ of the laser power. Shaded area corresponds to the laser power distributions for each TOF spectrum. Lower panel: Decomposition of the TOF spectrum for the average GMD value of 3639 channels into four Lorentzian curves: ${ }^{20} \mathrm{Ne}^{+}$from uncondensed gas and from the cluster, and ${ }^{22} \mathrm{Ne}^{+}$from uncondensed gas and from the cluster.

demonstrated in a previous paper [17].

In Fig. 2, the ${ }^{22} \mathrm{Ne}^{+}$yield is plotted against the laser power. The directly measured GMD signal is shown on the abscissa. It should be noted that the integrated intensity of the sharp peak exhibits a quadratic dependence on the laser intensity, indicating that the ionization of isolated atoms was due to the two-photon absorption in accordance with expectations. In contrast, the ion yields from the clusters show a linear dependence on the laser intensity. 


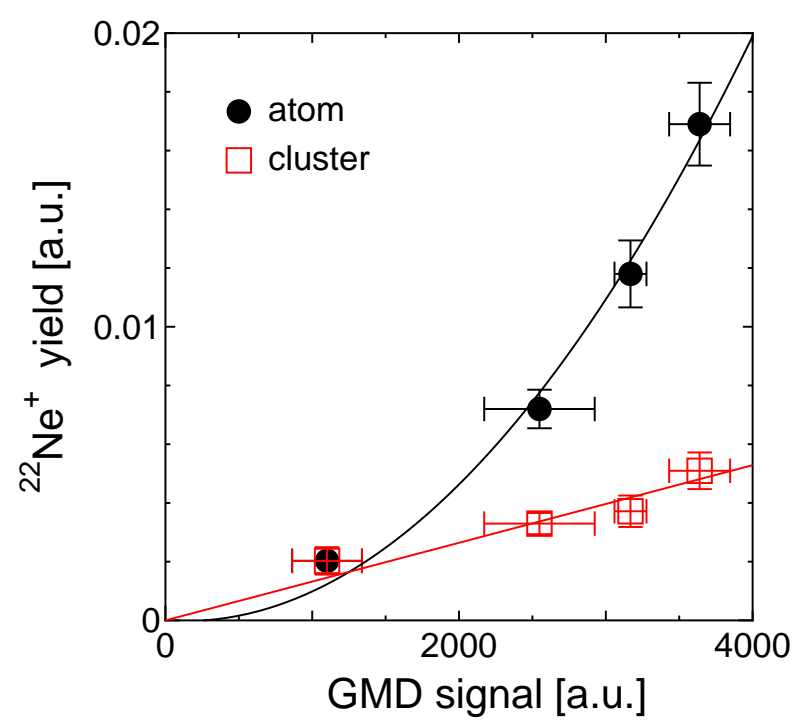

FIG. 2: ${ }^{22} \mathrm{Ne}^{+}$yield from uncondensed atoms (closed symbols) and from clusters (open symbols) as a function of the GMD channel. The intensities of these two components are re-normalized at the lowest point of the GMD scale. The horizontal error bars denote the standard deviation of GMD channels, and the vertical error bars denote the statistical errors. The line guides a quadratic dependence on the laser power for atoms and a linear dependence for clusters.

\section{DISCUSSIONS}

The present experimental results highlight that different kinds of "More is different" effects are induced in individual $\mathrm{Ne}$ atoms and in Ne clusters. The 2-photon ionization was observed for Ne atoms, while unexpected a linear dependence of ion yield on intensity was found for Ne clusters. This immediately raises several questions: "Why is 2-photon ionization not observed for the clusters?", "What is the mechanism of the linear dependence of ion yield on intensity?", "Is the linear dependence also observed in electron spectroscopy?" etc. Motivated by these questions, electron spectroscopic measurements have been conducted in our group using slightly higher photon energy $(20.3 \mathrm{eV})$ for larger clusters $\left(\mathrm{Ne}_{5000}\right)$. Since details of this works are beyond the scope of this paper, major conclusions drawn from these experiments are listed here:

- In the FEL intensity range from $\sim 10^{13}$ to $\sim 10^{14} \mathrm{~W} / \mathrm{cm}^{2}$, thermal electron emission, whose yield was proportional to the FEL intensity, was found. 
- The thermal electrons stem from the nano-plasma that is formed by an exciton-Mott transition (EMT) [4]. The thermal electron yield should be linearly proportional to the laser intensity, because the population of the intermediate excited states from which the nano-plasma is generated, is linearly proportional to the laser intensity.

- At sufficiently high FEL intensities $\left(\geq 10^{14} \mathrm{~W} / \mathrm{cm}^{2}\right)$, 2-photon ionization dominates even in the clusters.

- A novel ICD proposed by Kuleff et al. was observed on the insulating side of EMT, which corresponds to an FEL intensity of less than $10^{13} \mathrm{~W} / \mathrm{cm}^{2}$.

In the comparison of the present results of ion spectroscopy with those of the electron spectroscopy mentioned above, we should bear in mind that both the cluster size and the irradiation condition were different in the two experiments. Therefore, we give only a qualitative discussion here. Sugishima et al. [17] estimated how many charges were present before the Coulomb explosion by using a uniformly charged cluster model [15], and they concluded that about 50 charges were generated in the $\mathrm{Ne}_{1000}$ clusters. Because the estimated number of charged atoms, which should be smaller than that of excited atoms, is larger than the critical number for EMT, which is estimated at about 10 per 1000 atoms [18], we may conclude that our ion spectra have been recorded on the metallic side of EMT. On the other hand, since the FEL intensity is at most $10^{14} \mathrm{~W} / \mathrm{cm}^{2}$, the two-photon ionization was not a dominant ionization process. Thus the $I$-linear dependence of ion spectra is consistent with the results of electron spectroscopy.

\section{SUMMARY}

We carried out time-of-flight mass spectrometry for neon clusters that were exposed to intense free electron laser pulses with the wavelength of $62 \mathrm{~nm}$, which induce optical transition from the ground state $\left(2 \mathrm{~s}^{2} 2 \mathrm{p}^{6}\right)$ to an excited state $\left(2 \mathrm{~s}^{2} 2 \mathrm{p}^{5}\left(2 \mathrm{P}_{1 / 2,3 / 2}\right) 3 \mathrm{~d}\right)$ in a $\mathrm{Ne}$ atom. In contrast to isolated $\mathrm{Ne}$ atoms, in which a $\mathrm{Ne}^{+}$ion is produced by two-photon absorption, the $\mathrm{Ne}^{+}$ion yield from Ne clusters shows a linear dependence on the laser intensity. Motivated by these intriguing results, electron spectroscopic studies have been conducted in our group using slightly a higher photon energy $(20.3 \mathrm{eV})$ for larger clusters 
$\left(\mathrm{Ne}_{5000}\right)$. Combining the results of electron spectroscopy, we suggest that the linear intensity behavior is due to the nano-plasma that is formed as a result of an exciton-Mott transition.

\section{ACKNOWLEDGMENTS}

We thank A.I. Kuleff and L.S. Cederbaum for helpful discussion and for showing the manuscript prior to submission. We are grateful to the SCSS Test Accelerator Operation Group at RIKEN for continuous support in the course of the studies. We are also grateful to A. Belkacem and optics group in LBNL for fabricating the home-made focusing mirror. This work was supported by the X-ray Free Electron Laser Utilization Research Project of the Ministry of Education, Culture, Sports, Science and Technology of Japan (MEXT), by the Grant-in-Aid for the Global COE Program "The Next Generation of Physics, Spun from Universality and Emergence" from the MEXT, by the Grants-in-Aid (20310055, 21244062) from the Japan Society for the promotion of Science (JSPS), by the IMRAM project and by the MPG Advanced Study Group within CFEL. RD acknowledges support by DFG FOR 1789.

* Electronic address: nagaya@scphys.kyoto-u.ac.jp

[1] P.W. Anderson, Science 177 (1972) 393.

[2] For a recent review, V. Averbukh, Ph.V. Demekhin, P. Kolorenč, S. Scheit, S.D. Stoychev, A.I. Kuleff, Y.-C. Chiang, K. Gokhberg, S. Kopelke, N. Sisourat, and L.S. Cederbaum, J. Elec. Spectr. Rel. Phenom. 183 (2011) 36-47.

[3] L.S. Cederbaum, J. Zobeley, and F. Tarantelli, Phys. Rev. Lett. 79, 4778 (1997).

[4] N.F. Mott, Rev. Mod. Phys. 40,677 (1968).

[5] A.I. Kuleff, K. Gokhberg, S. Kopelke, and L.S. Cederbaum, Phys. Rev. Lett., 105, 043004 (2010).

[6] W. F. Chan, G. Cooper, X. Guo, and C. E. Brion, Phys. Rev. A 45, 1420 (1992).

[7] T. Shintake et al., Nature Photonics 2, 555 (2008).

[8] K. Motomura et al., J. Phys. B: At. Mol. Opt. Phys. 42, 221003 (2009).

[9] H. Fukuzawa et al., J. Phys. B: At. Mol. Opt. Phys. 42, 181001 (2009). 
[10] K. Nagaya et al., J. Electron Spectr. Related Phenom., 181 (2010) 125-128.

[11] O. F. Hagena and W. Obert, J. Chem. Phys. 56, 1793 (1972).

[12] R. Karnbach, M. Joppien, J. Stapelfeldt, J. Wörmer, and T. Möller, Rev. Sci. Instrum. 64, $2838(1993)$

[13] O. Jagutzki et al., IEEE Trans. Nucl. Sci. 49, 2477 (2002).

[14] K. Motomura et al.,, Nucl. Instrum. Meth. 606, 770 (2009).

[15] M.R. Islam, U. Saalmann, and J.M. Rost, Phys. Rev. A 73, 041201(R) (2006).

[16] T. Laarmann, A.R. B. de Castro, P. Gürtler, W. Laasch, J. Schulz, H. Wabnitz, and T. Möller, Phys. Rev. Lett. 92, 143401 (2004).

[17] A. Sugishima et al., Phys. Rev. A 86 (2012) 033203.

[18] S. Yase et al., in preparation. 